
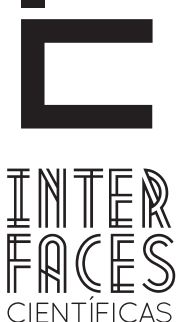

SAÚDE E AMBIENTE

\title{
AVALIAÇÃO DA DOENÇA PERIODONTAL EM ADULTOS NA POPULAÇ̃̃O QUILOMBOLA
}

Iara Ferreira Bruno

Jamille Alves Araújo Rosa"

\author{
Cláudia Moura de Melo" \\ Cristiane Costa da Cunha Oliveiralv
}

\section{RESUMO}

O objetivo deste estudo epidemiológico foi analisar a condição periodontal de população adulta quilombola sergipana. Foram avaliados 29 sujeitos pertencentes à comunidade na faixa etária de 35 a 44 anos. Foi realizado levantamento epidemiológico bucal com utilização de uma ficha clínica padronizada para investigar a ocorrência de doença periodontal utilizando o Índice Periodontal Comunitário (CPI) que permite avaliar a condição periodontal quanto à higidez, sangramento e presença de cálculo ou bolsa. A análise estatística foi conduzida com cálculo da prevalência da doença periodontal na faixa etária estudada, e teste qui-quadrado para verificar as diferenças significativas das relações pesquisadas.
Os resultados apontaram que entre os adultos da população quilombola do povoado Patioba, em Japaratuba-SE, Brasil, houve prevalência da doença periodontal, considerada alta $(75,86 \%)$, sendo maior no $1^{\circ}$ e $6^{\circ}$ sextantes bucais. Ressalta-se a necessidade de políticas de saúde com enfoque maior na promoção de saúde bucal para os quilombolas sergipanos.

\section{PALAVRAS-CHAVE}

Epidemiologia. Levantamentos de Saúde Bucal. Saúde de Grupos Populacionais Específicos. Doença Periodontal. 


\section{ABSTRACT}

The aim of this epidemiological study was to analyze the periodontal status of the adult quilombola population from Sergipe. It was appraised 29 subjects belonging to community aged 35-44 years. Oral epidemiological survey was conducted using a standardized case report form to investigate the occurrence of periodontal disease using the Community Periodontal Index (CPI) for assessing the periodontal condition regarding healthiness, bleeding and presence of calculus or purse. Statistical analysis was conducted for estimating the prevalence of periodontal disease in the age group studied and chi-square test to check for significant differences in the relationships studied. The results showed that among adult quilombola population from Patioba village, Japaratuba-SE, Brazil was the prevalence of periodontal disease, which is considered high (75.86\%), being higher in the 1st and 6th sextants mouth. One may emphasize the need for health policies with greater focus on oral health promotion for the quilombola of Sergipe.

\section{KEYWORDS}

Epidemiology. Oral Health Surveys. Health of Specific Population Groups. Periodontal Disease.

\section{INTRODUÇ̄̃̃O}

A saúde bucal é um importante aspecto da saúde das pessoas e de seu bem estar. Boa saúde bucal significa melhor convivência social, comunicabilidade, melhores condições de mastigação, que proporcionam prazer na degustação de diversos tipos de alimentos, autoconfiança social, ou seja, qualidade de vida. A maioria dos problemas bucais é passível de prevenção através de métodos há muito investigados. Quando a saúde bucal está compro-

\section{RESUMEN}

El objetivo de este estudio epidemiológico ha sido analizar el estado periodontal de la población adulta quilombola de Sergipe. Se evaluaron 29 sujetos de la comunidad de un grupo de edad de 35 a 44 años. Una encuesta epidemiológica bucal se llevó a cabo mediante un formulario clínico estandarizado para investigar la aparición de la enfermedad periodontal a través del Índice Periodontal Comunitario (CPI), que permite evaluar el estado periodontal en relación a la salubridad, el sangrado y la presencia de cálculo o bolsa. El análisis estadístico fue realizado para estimar la prevalencia de la enfermedad periodontal en el grupo de edad estudiado, y el test chi-cuadrado para verificar las diferencias significativas en las relaciones investigadas. Los resultados mostraron que entre la población adulta de la ciudad quilombola de la villa Patioba - Japaratuba - SE, Brasil hubo prevalencia de la enfermedad periodontal, considerada alta (75,86\%), siendo mayor en el $1^{\circ}$ y $6^{\circ}$ sextantes bucales. Se destaca la necesidad de las políticas de salud con un mayor enfoque en la promoción de la salud oral para los quilombolas de Sergipe.

\section{PALABRAS CLAVE}

Epidemiología. Encuestas de Salud Bucal. La salud de Grupos Poblacionales Específicos. Enfermedad Periodontal.

metida, o sofrimento ocasionado pelas doenças bucais ocorre em diversos setores da vida. Dor, desconforto, noites mal dormidas causadas por diversas patologias bucais, tais como a Cárie Dentária, a Doença Periodontal, as Erosões Dentárias, a Fluorose Dentária ou o Câncer Bucal, compromete a saúde e afetam inclusive a frequência às atividades escolares e laborativas, ou seja, a qualidade de vida fica comprometida ocasionando muitas despesas 
para a sociedade e para o indivíduo isoladamente (WATT, 2005).

A Doença Periodontal deve ser avaliada de forma minusciosa, pois além de ser uma razão forte para perda de dentes permanentes, há ainda a associação com as doenças sistêmicas, o que pode agravar ainda mais o comprometimento do elemento dentário, interferindo na resposta do hospedeiro à placa bacteriana.

As doenças periodontais podem se apresentar de duas formas: a reversível (gengivite) - quando a inflamação atinge apenas o rebordo gengival, os tecidos de proteção dentária e a irreversível (periodontite) - quando ocorre a perda de inserção dos tecidos periodontais que suportam e protegem o elemento dental (ligamentos periodontais, cemento radicular e osso alveolar), com formação de bolsa gengival, tendo assim uma difícil recuperação desses tecidos perdidos (ALVES et al., 2007).

Considerando que os sintomas odontoestomatológicos decorrentes das infecções derivam da interação entre a microbiota patógena e os mecanismos de defesa do hospedeiro acometido pela enfermidade, torna-se de suma relevância o estudo dos diferentes microorganismos compreendidos como agentes etiopatogênicos da Doença Periodontal em adultos. Dessa forma, os fatores irritantes locais, com destaque para o biofilme bacteriano, parecem desempenhar um papel fundamental no tocante à predisposição à instalação e progressão da Doença Periodontal (NOCITO-MENDOZA et al., 2003).
A Epidemiologia se empenha em compreender a distribuição de doenças ou agravos à saúde em coletividades humanas. Dessa forma, os epidemiologistas questionam se existe algum padrão na distribuição da doença no espaço, se há associação com alguma fonte etiológica (água, poluição atmosférica e violência urbana, por exemplo), se há evidência de transmissão e se há variação no tempo. Pessoas, lugar e tempo constituem a tríade básica da produção e interpretação dos constructos epidemiológicos, podendo ser escrita como pessoas em lugares/tempo, evidenciando o papel fundamental do espaço (físico e histórico) na ocorrência de eventos em saúde (AYRES, 2000).

Por isso, realizar um levantamento epidemiológico em saúde bucal é de grande importância, já que através de seus resultados é possível fazer um melhor planejamento das necessidades odontológicas de um bairro, cidade, estado e até mesmo de um país.

A necessidade de se entender a realidade sobre a condição de saúde bucal da população adulta quilombola em um povoado do interior do nordeste brasileiro será fundamental para que possibilitasse delinear planejamento das ações de saúde bucal que considere a necessidade desse grupo populacional.. Nesse contexto, o objetivo desse estudo foi determinar a prevalência e severidade da doença periodontal de indivíduos adultos em população Quilombola, do povoado de Patioba, em Japaratuba-SE.

\section{MATERIAL E MÉTODOS}

Trata-se de um estudo de campo observacional e transversal. 0 projeto foi encaminhado ao CEP da Universidade Tiradentes e aprovado sob número do protocolo 270610 , atendendo aos termos da resolução 196/96, de 10 de outubro de 1996 do Conselho Nacional de Saúde do Ministério de Saúde. 
A área de estudo foi o povoado Patioba, município de Japaratuba no Estado de Sergipe, Nordeste brasileiro. 0 espaço físico do povoado foi distribuído em ruas nomeadas como $A, B, C$ e D para facilitar o levantamento e em Patioba 2. Todas essas ruas foram igualmente selecionadas para a pesquisa. Essa distribuição foi usada como estratégia para identificar às residências da comunidade durante o desenvolvimento da pesquisa, visto que, não havia registro oficial dos logradouros existentes na comunidade.

Em 2009, a população desse povoado era composta de 593 habitantes, correspondendo a 186 famílias cadastradas no SIAB- Sistema de Informação da Atenção Básica (SIAB). Foram incluídos no estudo todos os indivíduos de 35 a 44 anos, de ambos os gêneros, que concordaram e assinaram o termo de consentimento livre e esclarecido, previamente lido e explicado. Foi utilizada a faixa etária de 35 a 44 anos, pois essa é a diretriz atual do Ministério da Saúde para levantamento epidemiológico de saúde bucal da populaçao adulta (BRASIL, 2010). Foram excluídos os hospitalizados. Após essa seleção, a amostra totalizou 29 adultos contando com $5 \%$ de possibilidade de perdas.

Foram realizados exames clínicos bucais executados de acordo com os critérios de diagnóstico do levantamento nacional de saúde bucal no ano de
2010 (BRASIL, 2010) para investigar a presença de lesões bucais, a ocorrência de doença periodontal utilizando o Índice Periodontal Comunitário (CPI) que permite avaliar a condição periodontal quanto à higidez, sangramento e presença de cálculo ou bolsa (OMS, 1999). O instrumento utilizado para coleta dos dados do levantamento bucal foi uma ficha clínica individual.

O levantamento foi conduzido por uma única examinadora calibrada, utilizando-se de ferramentas preconizadas pela OMS (1999): espátula (para auxílio de visualização do palato mole, unidades dentárias, funcionalidade lingual), luvas (para inspeção intra-oral). Considerou-se os critérios de diagnóstico do Projeto SB-2010 que faz parte de um processo histórico de diagnóstico da saúde bucal dos brasileiros demarcando o campo de atuação do componente de vigilância à saúde da Política Nacional de Saúde Bucal (BRASIL, 2010).

A análise estatística foi conduzida com cálculo das prevalências das doenças bucais na faixa etária estudada. Foi aplicado teste qui-quadrado para verificar a significância estatística das relações pesquisadas com nível de significância de 95\% $(p<0,05)$.

\section{RESULTADOS E DISCUSSÃO}

Dos 29 sujeitos adultos com faixa etária de 35 a 44 anos, $24,1 \%$ (7) foram do sexo masculino e $75,9 \%$ (22) do sexo feminino. A prevalência de doença periodontal foi de $75,86 \%(\mathrm{CP} \mid \neq 0)$, sendo maior no $1^{0}$ e $6^{\circ}$ sextante do $\mathrm{CPI}$, com percentual nulo de pessoas com $\mathrm{CPI}=0$. No $2^{\circ}$ sextante e no $6^{\circ}$ sextante não houve diferenças significativas nos estágios de severidade da doença periodontal. No primeiro sextante, a maioria significativa dos indivíduos se encontrava no estágio de gengivite $58,6 \%(p=0,04)$, outros em estágio de periodontite $13,8 \%$ e $27,6 \%$ possuíam o

sextante excluído. Houve presença de edentulismo em todos os sextantes principalmente no $1^{\circ}$ sextante $27,6 \%$ (8) e no $2^{\circ}$ sextante $31 \%$ (9), sendo este o maior percentual do código representativo de sextante excluído (Tabela 1).

No presente estudo a prevalência da doença periodontal na população adulta quilombola, na zona rural do municipio nordestino foi de $75,86 \%$ considerada alta, sendo mais prevalente no $1^{\circ}$ e $6^{\circ}$ sextantes. A maioria dos indivíduos se encontramno está- 
gio de gengivite principalmente quando se considera $1^{\circ}, 3^{\circ}, 4^{\circ}$ e $5^{\circ}$ sextantes. A divisão em sextantes, de acordo com o modelo do último Levantamento Epidemiológico Nacional (BRASIL, 2010), permite ter uma visão adequada da severidade doença nos diversos sítios, sendo esse um diferencial desta pesquisa, considerando a maioria dos estudos anteriores que não demonstram esssa ocorrência por sextantes.

O estudo de Macêdo e outros (2006) em uma área rural do Estado da Bahia, no nordeste brasileiro, estimaram a prevalência e os fatores associados à periodontite em 172 indivíduos, de 20 a 60 anos de idade residentes no povoado de Matinha dos Pretos, Feira de Santana (BA). A gengivite foi observada em $97,7 \%$ da população, correspondente à percent agem de indivíduos com presença de sangramento à sondagem. A periodontite foi observado em $24,4 \%$ da população.
Carvalho et al (2010) em revisão crítica da literatura internacional dos últimos dez anos verificaram que as doenças bucais, com presença de cálculo e boslas rasas tem prevalecido na população adulta e que a maioria na faixa etária de 35 a 44 anos. No entanto sabe-se que no Brasil e especialmente em populações rurais essa situação é mais severa e tem apresentado níveis importantes de edentulismo como o estudo de Macedo et al (2006) que encontrou cinquenta por cento (50\%) do população rural baiana com mais de 4 dentes perdidos.

No presente estudo, em todos os sextantes pesquisados, houve presença de edentulismo, o que aponta para existência de perdas dentárias evidentes. A maior porcentagem do código representativo de sextante excluído ocorreu no $2^{\circ}$ sextante. $01^{\circ}$ sextante obteve importância secundária nesse quesito. 0 fato de haver maior perda nos sextantes da arcada dentá-

Tabela 1- Distribuição de frequência dos escores do índice CPI nos sextantes da arcada superior e inferior dos indivíduos adultos quilombolas de Patioba/SE - 2010/2011.

\begin{tabular}{|c|c|c|c|c|c|c|c|c|c|c|c|c|c|}
\hline \multirow[b]{3}{*}{ Sextantes } & \multicolumn{13}{|c|}{ Índice CPI } \\
\hline & \multicolumn{2}{|c|}{$\mathrm{CPI}=0$} & \multicolumn{2}{|c|}{$\mathrm{CPI}=1$} & \multicolumn{2}{|c|}{$\mathrm{CPI}=2$} & \multicolumn{2}{|c|}{$\mathrm{CPI}=3$} & \multicolumn{2}{|c|}{$\mathrm{CPI}=4$} & \multicolumn{2}{|c|}{$C P I=X$} & \multirow[b]{2}{*}{$P$} \\
\hline & $N$ & $\%$ & $\mathrm{~N}$ & $\%$ & $\mathrm{~N}$ & $\%$ & $\mathrm{~N}$ & $\%$ & $\mathrm{~N}$ & $\%$ & $\mathrm{~N}$ & $\%$ & \\
\hline $1^{0}$ & 0 & 0 & 4 & 13,8 & 13 & 44,8 & 2 & 6,9 & 2 & 6,9 & 8 & 27,6 & 0,04 \\
\hline $2^{\circ}$ & 3 & 10,3 & 6 & 20,7 & 8 & 27,6 & 3 & 10,3 & 0 & 0 & 9 & 31 & 0,25 \\
\hline $3^{0}$ & 2 & 6,9 & 2 & 6,9 & 13 & 44,8 & 4 & 13,8 & 0 & 0 & 8 & 27,6 & 0,04 \\
\hline $4^{0}$ & 1 & 3,4 & 3 & 10,3 & 17 & 58,6 & 2 & 6,9 & 1 & 3,4 & 5 & 17,2 & 0,00 \\
\hline $5^{\circ}$ & 1 & 3,4 & 1 & 3,4 & 21 & 72,4 & 4 & 13,8 & 1 & 3,4 & 1 & 3,4 & 0,00 \\
\hline $6^{\circ}$ & 0 & 0 & 4 & 13,8 & 13 & 44,8 & 4 & 13,8 & 1 & 3,4 & 7 & 24,1 & 0,06 \\
\hline \multicolumn{14}{|c|}{ Total dos pesquisados: $\mathrm{n}=29$} \\
\hline \multicolumn{14}{|c|}{$\begin{array}{l}\text { OBS: (0) Saúde gengival } \\
\text { (1) Sangramento a sondagem } \\
\text { (2) Presença de cálculo com ou sem sangramento, excesso ou falta de material restaurador } \\
\text { (3) Profundidade de sondagem maior que } 3,5 \mathrm{~mm} \text { e menor que } 5,5 \mathrm{~mm} \\
\text { (4) Profundidade de sondagem ultrapassa } 5,5 \mathrm{~mm} \\
\text { (X)Sextante excluído }\end{array}$} \\
\hline
\end{tabular}


ria superior pode sugerir dificuldades maiores de higiene bucal nesses sítios.

O estudo de Saliba et al (2010) em população rural de São Paulo, apontou que a perda dentária se iniciava precocemente e progredia com o avançar da idade. Foi salientado que os serviços oferecidos ainda não eram capazes de limitar os danos causados pelas doenças bucais. O Levantamento Nacional de Saúde Bucal SB Brasil 2010 demonstrou a condição de saúde bucal da população adulta brasileira com relação a doença periodontal. As informações coletadas indicaram que as formas mais graves ocorreram de modo significativo nos adultos (35 a 44 anos), nos quais se observou uma prevalência de 19\% (BRASIL, 2011).

Freitas et al (2011), em revisão sobre saúde da população quilombola, já haviam comentado sobre a existência de uma grande disparidade na Atenção à Saúde no Brasil, havendo necessidade de comprometimento social e profissional a esses grupos ainda excluídos

Neste estudo a presença de edentulismo na população quilombola adulta na zona rural, pode sugerir a existência de deficiência nos programas de saúde bucal destinados a população jovem e adulta, já que segundo as metas da OMS para 2010, para a faixa etária de 35 a 44 anos deveria haver $90 \%$ com 20 ou mais dentes presentes na boca (BRASIL, 2011).

Diante do exposto vale ressaltar a importância de se planejar políticas públicas específicas para a população quilombola adulta do município de Japaratuba em Sergipe. Outros estudos devem seguir na mesma linha buscando fatores que possam influenciar na prevalência e progressão da doença em adultos quilombolas do estado de Sergipe.

\section{CONCLUSÃO}

A prevalência da doença periodontal na população estudada foi considerada alta $(75,86 \%)$, observada na maioria dos sextantes pesquisados, sendo que o $5^{\circ}$ sextante teve a maior prevalência
(93,1\%). Os sextantes excluídos, principalmente na arcada superior, apontam para o edentulismo parcial presente na população adulta quilombola pesquisada.

\section{REFERÊNCIAS}

ALVES, C.; ANDION, J.; BRANDÃO, M.; MENEZES. R. Mecanismos patogênicos da doença periodontal associada ao Diabetes Melito. Arquivos Brasileiros de Endocrinologia \& Metabólogia, São Paulo, v.51, n.7, p.1050-105, 2007.

AYRES JR. Debate sobre o artigo de Dina Czeresnia \& Adriana Maria Ribeiro. Caderno de Saúde Pública, BRASIL. Ministério da saúde. SB Brasil, Pesquisa Nacional de Saúde Bucal, manual de calibração de examinadores, Brasília DF, 2010.

Brasil. Ministério da saúde. Projeto SB Brasil 2010: condições de saúde bucal da população brasileira 2010-2011. Resultados principais. Brasília-DF, 2011. v.16, p. 610-611, 2000. 
CARVALHO, E.S.; BASTOS, R..S; RODRIGUES, AD.M.; MELLO, W.M;. LAURIS, J.R.P.; BASTOS, J..RM.; SALES PERES, S..HC. Epidemiologia das doenças bucais em indivíduos na faixa etária entre 35 e 44 anos: o cenário epidemiológico do trabalhador. Revista Gaúcha de Odontologia, Porto Alegre, v.58, v.1, p. 109-114, 2010.

FREITAS, D.A.; CABALLERO, A.D.; MARQUES, A.S.; HERNANDÉZ, C.I.V.; ANTUNES, S..LN.O. Saúde e comunidades quilombolas: uma revisão de literatura. Revista CEFAC (online), v,13, n.5,p. 937-943, 2011.

MACÊDO, T.C.N.; COSTA, M.C.N.; GOMES-FILHO, I.S.; VIANNA, M.I.P.; SANTOS, C.T. Factors related to periodontal disease in a rural population. Brazilian. Oral Research [online], 2006; 20 (3): 257-262.

NOCITO-MENDOZA, I.; VASCONI-CORREAS, M.D.; PONCE DE LEÓN-HORIANSKI, P.; ZDERO-PANDZICH, M.. Entamoeba gengivalis and Trichomonas tenax in diabetic patients. Revista del ilustre consejo General de Colegios de Odontólogos y Estomatólogos de España,. v. 8, n.1, p. 13-23, 2003.

Recebido em: 1 nov. 2012

Avaliado em: 28 nov. 2012

Aceito em: 10 dez. 2012
ORGANIZAÇÃO MUNDIAL DE SAÚDE. Levantamento epidemiológico básico de saúde bucal, manual de instruções. 4. Ed. São Paulo: Santos, 1999. 66p.

SALIBA, N.A.; MOIMAZ, S.A.S.; SALIBA, O.; TIANO, A..V.P. Perda dentária em uma população rural e as metas estabelecidas pela Organização Mundial de Saúde. Ciência \&. Saúde Coletiva [online]. 2010; 15(10): 1857-1864.

WATT, R.G. Strategies and approaches in oral disease prevention and health promotion. Bull World Health Organization, v.83, n,9, p.711-8, 2005.

Acadêmica do curso de Odontologia da Universidade Tiradentes - Unit/SE.

II Graduada em Odontologia; especialista em Saúde Pública pela Universidade Estácio de Sá; mestre em Saúde e Ambiente pela Universidade Tiradentes - Unit/SE.

III Bacharel em Biologia pela Universidade Federal de Viçosa; doutora em Parasitologia; professora titular III da Universidade Tiradentes; pesquisadora do Instituto de Tecnologia e Pesquisa - ITP/SE (coordenadora do Laboratório de Biologia Molecular).

IV Graduada em Odontologia; doutora em Odontologia (Saúde Coletiva); professora titular da Universidade Tiradentes; pesquisadora do Instituto de Tecnologia e Pesquisa - ITP/SE. 